\title{
Investigating physical and nutritional changes during prolonged intermittent fasting in hemodialysis patients: a prospective cohort study
}

\begin{abstract}
Objective: Studies investigating the health effects of prolonged intermittent fasting during Ramadan among Muslim patients on hemodialysis (HD) are limited and reported heterogeneous findings. This study aimed to evaluate the effects of intermittent fasting during Ramadan on nutritional and functional status of patients on maintenance HD. Design and methods: This was a 12-week, multicenter, prospective observational study. The study setting included three HD centers. Adult Muslim patients, who were undergoing HD session thrice weekly and planned to fast during Ramadan, were screened for eligibility and recruited. Nutritional and functional status assessments were carried out 2 weeks before (V0), at the fourth week of Ramadan (V1), and 4 weeks after Ramadan (V2). Nutritional status parameters included anthropometry (body mass index, interdialytic weight gain, waist circumference), body composition (mid-arm circumference, triceps skinfold, body fat percentage), blood biochemistry (albumin, renal profile, lipid profile, and inflammatory markers), blood pressure, dietary intake, and handgrip strength. Changes in nutritional and functional status parameters across study timepoints were analyzed using repeated-measures analysis of variance. Results: A total of 87 patients completed the study, with 68 patients ( $78.2 \%)$ reporting fasting $\geq 20$ days. Ramadan fasting led to significant reductions (all $\mathrm{P}<.05$ ) in body mass index, interdialytic weight gain, waist circumference, mid-arm circumference, fat tissue mass, and body fat percentage, but these were not accompanied by any significant change in lean tissue mass $(\mathrm{P}>$ .05). Significant improvement was observed in serum phosphate levels, but serum albumin, urea, and creatinine were also reduced significantly during Ramadan $(\mathrm{P}<.05)$. There were no significant changes in lipid profile and inflammatory markers. Interestingly, energy and protein intakes remain unchanged during Ramadan. Handgrip strength improved significantly during Ramadan and further improved after Ramadan. Conclusion: Intermittent Ramadan fasting leads to temporary changes in nutritional status parameters and poses nondetrimental nutritional risk for patients on maintenance HD.
\end{abstract}

